\title{
LETTER
}

\section{Zemax ray tracing model for plasma waveguides}

\section{Recent citations}

- Direct refractive index retrieval from
$\frac{\text { interferometry measurements }}{\text { Mario Galletti et al }}$
- Plasma density profile measurements for
$\frac{\text { ultra-short high power laser beam quiding }}{\text { experiments at SPARC LAB }}$
G Costa et al

View the article online for updates and enhancements.

\section{IOP ebooks}

Bringing together innovative digital publishing with leading authors from the global scientific community. Start exploring the collection-download the first chapter of every title for free. 


\title{
Letter
}

\section{Zemax ray tracing model for plasma waveguides}

\author{
F Bisesto ${ }^{1}$, M Galletti $^{2,3}$ and A Curcio ${ }^{4}$ \\ 1 INFN-LNF, Via Enrico Fermi 40, 00044 Frascati, Italy \\ ${ }^{2}$ GoLP Instituto de Plasmas e Fusão Nuclear, Instituto Superior Tecnico, Universidade de Lisboa, \\ Av. Rovisco Pais 1049-001 Lisbon, Portugal \\ ${ }^{3}$ Central Laser Facility, Science and Technology Facilities Council, Rutherford Appleton Laboratory, \\ Harwell Science and Innovation Campus, Didcot OX11 0QX, United Kingdom \\ ${ }^{4}$ CERN: Conseil Européen pour la Recherche Nucléaire, Geneva, Switzerland \\ E-mail: fabrizio.giuseppe.bisesto@lnf.infn.it
}

Received 24 August 2020

Accepted for publication 10 January 2020

Published 28 January 2020

\begin{abstract}
Plasma-based waveguides are currently employed for laser wakefield acceleration to extend the focal region of laser beams. A parabolic transverse plasma density profile, suitable for laser guiding, can be established in a dielectric capillary as a consequence of a discharge in gas. In this work, we report on a new ray tracing model, based on the Zemax software, able to simulate the envelope of a laser beam propagating through a plasma waveguide. The potentialities offered by Zemax in terms of geometries and materials and the possibility to create custom objects do allow the simulation of complex and various scenarios, as for example curved plasma waveguides.
\end{abstract}

Keywords: laser, plasma, laser-wakefield acceleration (LWFA), waveguide

(Some figures may appear in colour only in the online journal)

\section{Introduction}

Gas-filled dielectric capillaries are becoming a very important components for the next-generation plasma wakefield accelerators. Indeed, they are employed for plasma channels generation via electric discharges in gas. Applying a voltage of the order of tens $\mathrm{kV}$ to the electrodes at the extremities of a few cm-long capillary, it is possible to induce gas-ionization, e.g. in light gases as hydrogen or helium, achieving electron densities within a range which is useful for plasma acceleration over centimeter scales, i.e. in the order of $10^{17}-10^{18} \mathrm{~cm}^{-3}$. Plasma wakefields in dielectric capillaries have been studied in the last two decades both for beam and laser driven plasma acceleration [1-3]. One application of plasma discharge capillaries is that to use one (or more) electron beams for driving a plasma wakefield which establish an accelerating field for a following witness beam. Plasma discharge capillary is also used to exploit the huge magnetic field generated by the discharge current (up to tens of times larger than what provided by permanent quadrupoles) to tightly focus charged particle beam on very short distance [4-7]. Another important application of plasma channels in dielectric structures can be found in laser-wakefield acceleration (LWFA). Indeed, after a characteristic time of heat exchange between the plasma and the capillary walls, the transverse plasma density profile can acquire a parabolic trend, with a minimum at the center of the capillary, i.e. on its symmetry axis. A generic functional expression for the plasma refractive index would be given by $n(r)=\left(1-\omega_{p}(r) /\left(\gamma \cdot \omega_{0}\right)\right)^{1 / 2}$, where $r$ is the radial coordinate, $\omega_{p}$ is the plasma frequency, $\omega_{0}$ is the laser frequency and $\gamma=\left(1+a^{2} / 2\right)^{1 / 2}$ is the Lorentz factor, taking into account for the electron relativistic quivering through the normalized laser intensity $a$ [8]. It has been already shown that plasmas with parabolic transverse profiles can act as waveguides [913]. In this case, the associated refractive index is such that 


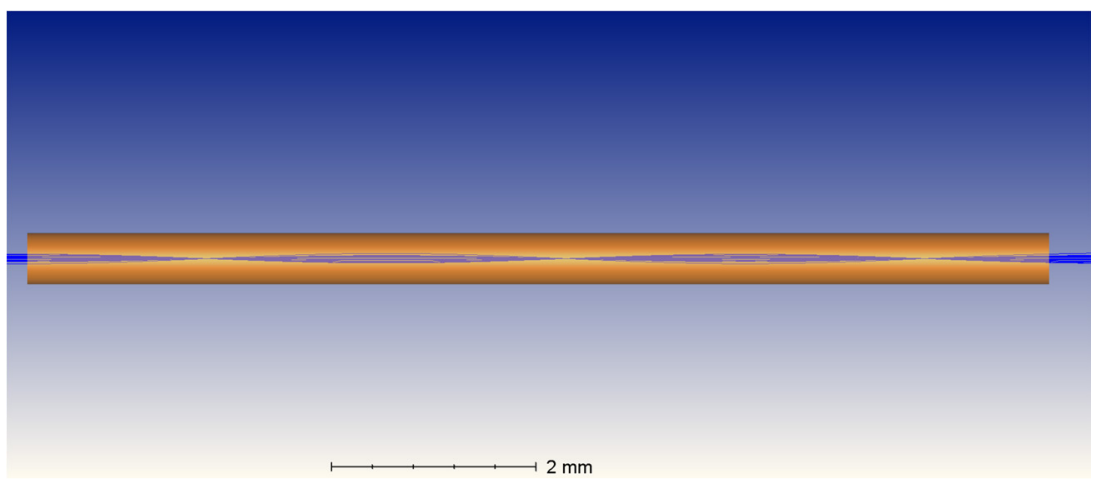

Figure 1. Ray propagation along the plasma waveguide axis. The ray envelope shows the typical oscillating behaviour of a guided beam.

it can overcome the natural divergence of a properly matched laser beam, keeping it focused over several Rayleigh lengths. In detail, a laser beam is considered matched into a plasma channel if $w_{0}=r_{m}$, where $w_{0}$ is the beam waist at the entrance of the channel and $r_{m}$ is the radius of the guiding channel itself. This feature represents a very powerful tool for laser driven plasma accelerators, where the limit in the acceleration length due to the laser diffraction is one of the most critical. Several experiments $[11,14]$ have been performed using plasma channels also as waveguides, extending the effective acceleration length and leading to the generation of $\mathrm{GeV}$-scale electron beams [15-18]. Similarly to any conventional particle accelerator component, it is important to well-characterize plasma waveguides [19]. One of the most diffused techniques for plasma density measurements is that to use an optical interferometer [20-23]. This method is largely employed for plasmas generated from supersonic gas-jets. The implementation of a Abel-inversion makes then possible the retrieval of plasma density profiles. On the other hand, for the gas-filled capillaries one has to take into account also for the outer dielectric structure that can introduce optical distortions of different kind. In this sense, the possibility to optically simulate such a structure can represent a very useful tool. Zemax is a raytracing software able to simulate the propagation of light trough different materials and geometries. Moreover, it allows to define custom objects being very attractive for scientific purposes like in $[24,25]$. In this work, we propose a raytracing model, based on a custom Zemax [26] dynamic-link library (DLL) which defines a waveguide starting from a parabolic plasma transverse density profile. It can model the propagation of a low intensity laser beam, i.e. $a \ll 1$ and $\gamma \sim 1$, in such a plasma channel inside a capillary. In detail, for laser power $P<P_{c} \simeq 17\left(\omega / \omega_{p}\right) \mathrm{GW}$, where $\omega_{p}$ is the plasma frequency, self focusing does not affect the surrounding plasma and the beam propagation as well. Althought this limitation, our model can be properly used to study laser propagation for linear regime LWFA, e.g. plasma wave exitation for external injection experiments [27, 28], and related diagnostics, usually employing low power probe beams. At this regard, we have realized an ideal Mach-Zehnder interferometer experiment in the Zemax environment, analyzing the interferograms a posteriori with a Abel-inversion software and retrieving the starting plasma profile to benchmark our model of plasma profile. Finally, we have pushed the potentiality of Zemax for studying the light propagation in a curved plasma-discharge capillary, interesting for a wide range of applications both in optics in general, and in laser plasma acceleration in particular [29-31].

\section{Zemax model}

Zemax editor provides two different scenarios, sequential (SC) and non-sequential (NSC) modes. In the first one, it is possible to design a setup by creating items, e.g. lenses, apertures, polarizers etc, as a succession of consecutive surfaces along the ray propagation axis. On the other hand, in the NSC scenario, one can place objects wherever in any point of the three-dimensional space. In this way, it is possible to design almost every kind of optical setup, as for example an interferometer. Once the layout has been completed and a light source has been activated, Zemax ray-tracing starts propagating each ray through the optical system. Placing a detector in the desired location, the user can visualize the ray position as propagated by Zemax. Therefore, only a geometrical optics analysis is possible, while the propagation in physical optics is reserved only to the SC mode. Nevertheless, both scenarios allow the user to customize his own setup, as for example defining a dispersion curve for a new material, designing a particular object geometry and programming special DLL for more advanced features. For our aim, working in the NSC mode, we wrote a custom DLL to define a parabolic refractive index profile, like for a gradient-index (GRIN) structure, starting from plasma parameters. The chosen electron density profile has been $n_{e}(r)=n_{0}+\left(\Delta n / r_{m}^{2}\right) r^{2}$, with $n_{0}$ the on-axis density, $\Delta n$ the density variation on the transverse plane of the capillary and $r_{m}$ the capillary radius. Such a DLL can be used to define the refractive index of any kind of object. In this work, we have limited our study to cylindrical volumes, straight and curved.

\section{Benchmarking}

\subsection{Interferometric measurement}

To test the goodness of our model, firstly we have tested the longitudinal propagation of a matched laser beam through a plasma waveguide defined by $n_{0}=1 \times 10^{18} \mathrm{~cm}^{-3}$, 


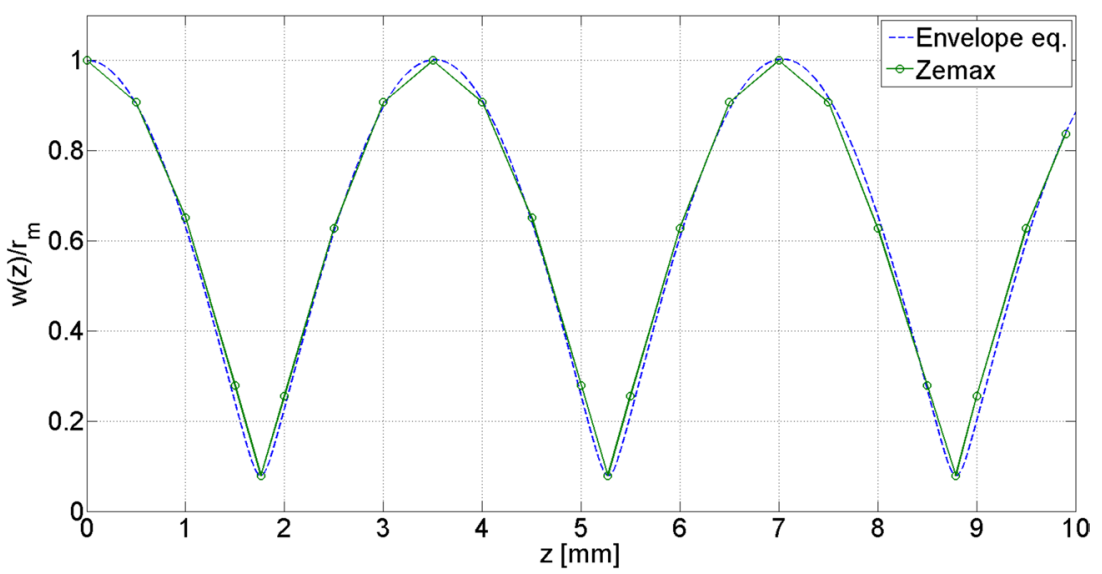

Figure 2. Comparison between numerical solution of the envelope equation given by equation (1) (dashed lined) and Zemax simulation result (solid line), showing an excellent agreement.

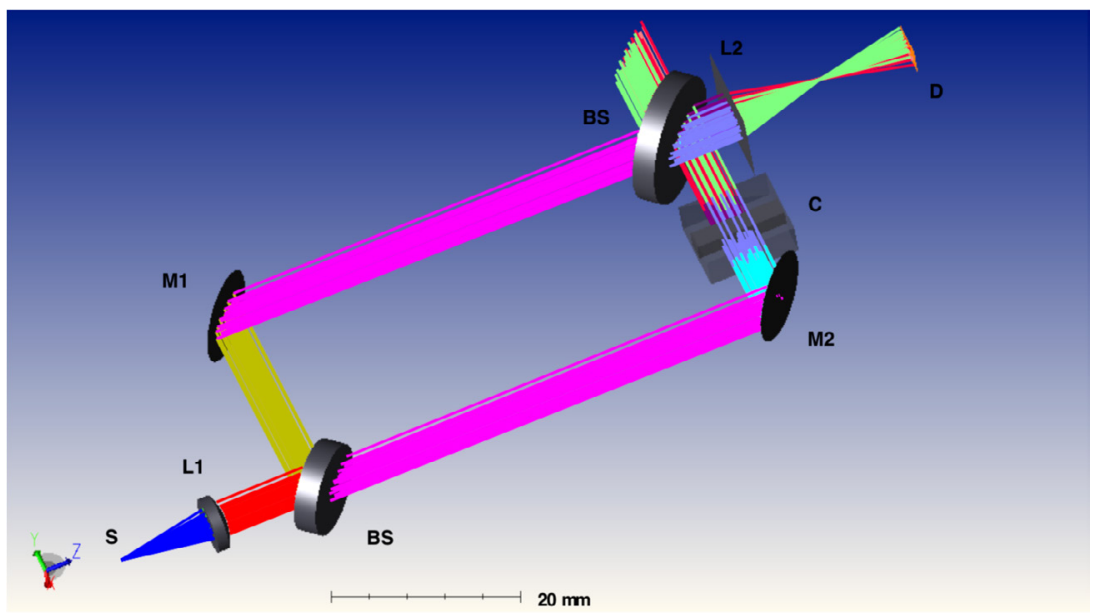

Figure 3. Zemax shaded model of setup layout. A point source (S) is collimated by a lens (L1) to be used as optical probe into the interferometer. A first beam splitter (BS) makes two replica of the beam, one acting as reference and the other illuminating the capillary (C). A second BS merges the two beams and a lens (L2) images them on a rectangular detector (D), $4 \times 4$ mm with $2000 \times 2000$ pixels, resulting in $2 \mu \mathrm{m}$ resolution.

$\Delta n=5 \times 10^{18} \mathrm{~cm}^{-3}$ and $r_{m}=60 \mu \mathrm{m}$. Figure 1 shows the propagation along the capillary axis of a ray fan, starting with a Gaussian transverse distribution matched with the plasma waveguide. Placing different detectors along the capillary to measure the beam radius, we have compared our results with the numerical solution of the envelope equation given by [32]

$$
\frac{d^{2} R}{d z^{2}}=\frac{1}{z_{R}^{2} R^{3}}\left(1-\frac{\Delta n}{\Delta n_{c}} R^{4}\right)
$$

where $R=w(z) / r_{m}, z_{R}=\pi r_{m}^{2} / \lambda$ and $\Delta n_{c}=\left(\pi r_{m}^{2} r_{e}\right)^{-1}$. The quantity $w(z)$ represents the beam width at the $1 / e^{2}$ fall of the light intensity profile, $\lambda=800 \mathrm{~nm}$ is the laser wavelength and $r_{e}$ the classical electron radius. Figure 2 shows the comparison between the envelope equation solution and the Zemax simulation. As it is possible to see, they are in excellent agreement, confirming a proper definition of the plasma waveguide in the Zemax custom library. Figure 3 shows the Zemax shaded model of a setup layout. Here, a Mach-Zehnder interferometer has been designed, using a collimated ray source at $800 \mathrm{~nm}$ wavelength as optical probe. Along one arm, a dielectric capillary, defined as a hollow channel inside a parallelepipedic

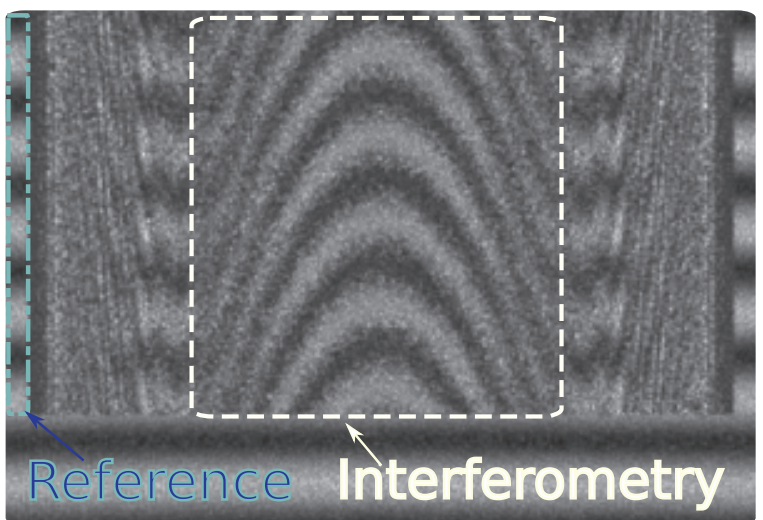

Figure 4. Interferogram simulated by Zemax using $1 \times 10^{6}$ rays.

silica structure, has been placed with its axis orthogonal to the rays. These, in turn, are collected by a $f=10 \mathrm{~cm}$ lens, making a 1:1 image of the capillary longitudinal plane onto a rectangular detector, $4 \times 4 \mathrm{~mm}$ with $2000 \times 2000$ pixels. The second arm, where rays propagate in vacuum, is used as reference beam. The plasma parameters used in this case are 


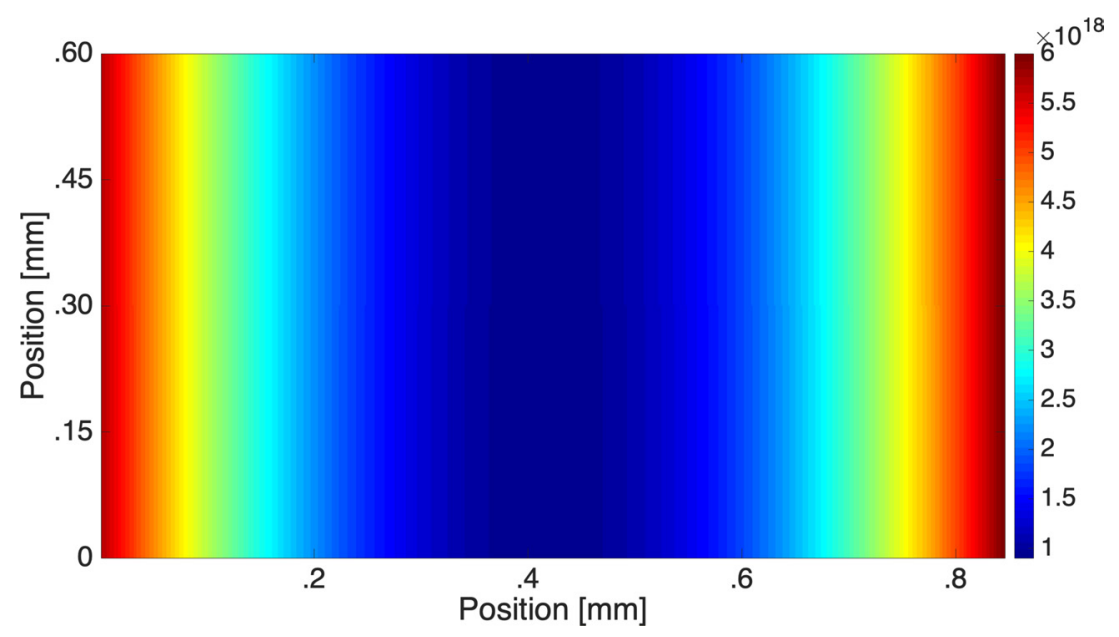

Figure 5. Electron density map retrieved from the interferogram shown in figure 4.



Figure 6. Line profile of figure 5 showing the transverse electron plasma density, consistent with the input parameters defining the Zemax simulation. The parameters $n_{0}=1 \times 10^{18} \mathrm{~cm}^{-3}, \Delta n=5 \times 10^{18} \mathrm{~cm}^{-3}$ and $r_{m}=500 \mu \mathrm{m}$ have been retrieved.

$n_{0}=1 \times 10^{18} \mathrm{~cm}^{-3}, \Delta n=5 \times 10^{18} \mathrm{~cm}^{-3}$ and $r_{m}=500 \mu \mathrm{m}$. Figure 4 shows a simulated interferogram. This has been obtained using a source made by $1 \times 10^{6}$ rays illuminating the sample. Since only a portion of capillary is placed along the beam, the interferogram presents also straight lines on both sides and on the bottom, defining the background signal and acting as reference for the analysis. These fringes are due to a $0.1^{\circ}$ horizontal misalignment of mirror M1. Data provided by Zemax have been processed by means of an external analysis tool.

The interferometry code we have used is made up by two main parts: the raw data manipulation and the $2 \mathrm{D}$ analysis. In the data manipulation section, the routine extrapolate, from the raw interferogram trace, the induced phase shift calculating the bending of the fringes, $\Delta K$, respect to the reference ones. The phase shift $\phi$ results finally in

$$
\Delta K=\frac{\phi}{2 \pi} .
$$

Successively, in the 2D analysis section the routine numerically calculates the gradient of the induced phase $\nabla \phi$ map. Considering the following general well-known relationships:

$$
\begin{gathered}
N(\mathbf{x}, \mathbf{y})-N_{0} \propto \alpha(\mathbf{y}) \int \nabla \phi F(\mathbf{x}) d \mathbf{x} \\
T(\mathbf{x}, \mathbf{y})=\frac{N_{0}-1}{N(\mathbf{x}, \mathbf{y})-1} T_{0}
\end{gathered}
$$

where $\alpha(\mathbf{y})$ and $F(\mathbf{x})$ are respectively a constant taking into account the boundary condition and a form factor taking into account the geometry, $T_{0}$ and $N_{0}$ are, respectively, the room temperature and the index of refraction relative to it, the routine numerically extrapolates the $2 \mathrm{D}$ density/temperature map. However, for the purposes of the present work we have been only interested in the density profile.

Figure 5 shows the result of the analysis of figure 4, reporting the corresponding 2D electron plasma density map, where the density distribution of the plasma waveguide is recovered. Figure 6 shows the data extrapolated from a central row of figure 5. This plot represents the retrieved density profile due to plasma-induced fringe shift. The resulting curve well-reproduce the parabolic profile defined as input in the Zemax object modeling the plasma waveguide, i.e. $n_{e}(r)\left[\mathrm{cm}^{-3}\right]=1 \times 10^{18}+5 \times 10^{18} \cdot r[\mu \mathrm{m}]^{2} / 500^{2}$. 


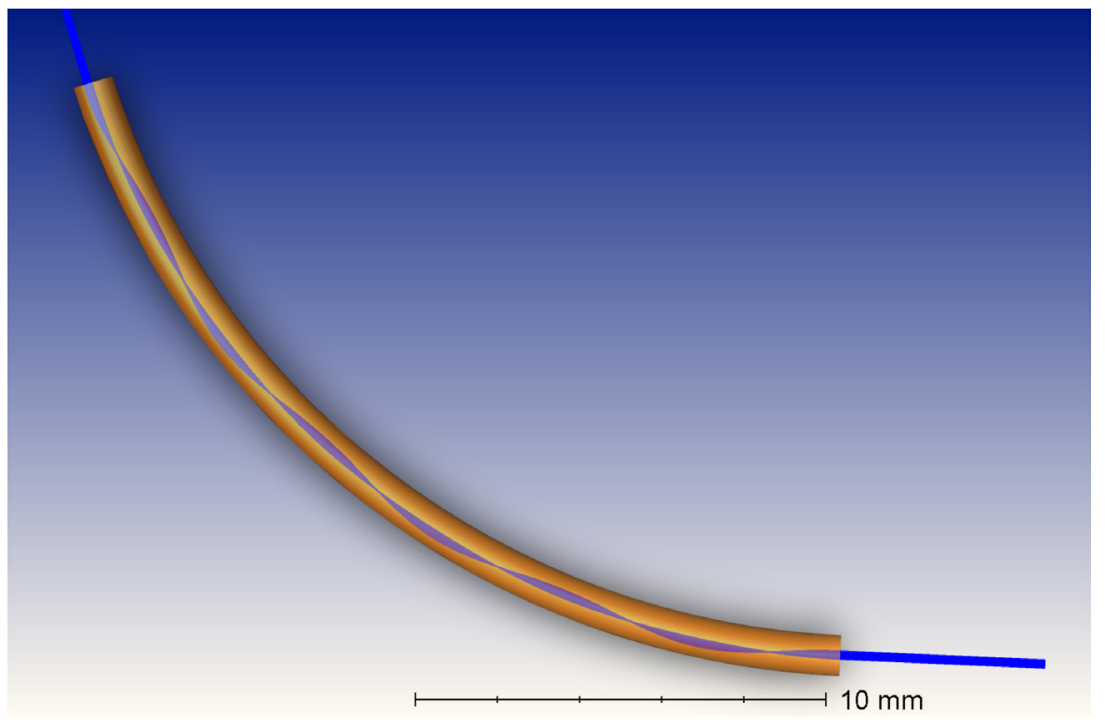

Figure 7. Rays propagation inside a curved capillary. A parabolic plasma profile has been defined using the following parameters: $n_{0}=1 \times 10^{18} \mathrm{~cm}^{-3}, \Delta n=5 \times 10^{18} \mathrm{~cm}^{-3}$ and $r_{m}=60 \mu \mathrm{m}$.

\subsection{Laser propagation in a curved capillary}

After having performed these benchmarks, we have exploited one of the main features offered by Zemax, consisting in the possibility to define different object geometries. Thus, we have studied a curved plasma waveguide. For this aim, we have used a torus volume object in Zemax adapting our DLL to this case. Figure 7 shows the propagation of a matched ray fan inside such a structure, as done for figure 1 . The capillary has a curvature radius $R_{c}=20 \mathrm{~mm}$ and a length equals to $25 \mathrm{~mm}$. As for the straight capillary simulation, the parabolic plasma profile employed has been defined by $n_{0}=1 \times 10^{18}$ $\mathrm{cm}^{-3}, \Delta n=5 \times 10^{18} \mathrm{~cm}^{-3}$ and $r_{m}=60 \mu \mathrm{m}$. Also in this situation, the model has well-reproduced the guiding effect due to the plasma channel, taking into account also for the bent structure of the dielectric capillary. The hard challenge of studying toroidal shapes of plasma waveguides has been anticipated in [33], here solved via the exploitation of our powerful and custom DDL for Zemax.

Furthermore, the use of detectors along the curved capillary allows for reconstructing the field profile $E(r)$ of the guided beam at any chosen cross-section of the plasma waveguide. Indeed, by an expansion over electromagnetic hybrid modes $E H_{1 n}[3,34]$, say $E(r)=\Sigma_{n} c_{n} E H_{1 n}$, it becomes possible studying the excited modes together with the corresponding amplitudes $c_{n}$ :

$$
c_{n}=\frac{\int_{0}^{1} E(x) J_{0}\left(u_{n} x\right) d x}{\int_{0}^{1} J_{0}\left(u_{n} x\right) d x}
$$

where the normalized integration variable is $x=r / r_{m}$ and $\left|c_{n}\right|^{2}$ indicates the energy stored in each mode $n$.

\section{Conclusion}

In conclusion, we have reported on a new ray tracing model based on the Zemax software that is able to reproduce the behaviour of a plasma waveguide, commonly used to guide a laser in LWFA experiments, realized by means of a gas discharge inside a dielectric capillary, for a low intensity laser beam $\left(a_{0} \ll 1\right)$. Starting from the plasma parameters defining the transverse electron plasma density profile, we have exploited a custom DLL for calculating the correspondent refractive index profile, responsible for light guiding. To test the goodness of our model, firstly we have injected a ray fan through a straight plasma channel and we have observed its behaviour. In particular, placing several detectors along the capillary, we have been able to compare the simulated beam size with respect to that evaluated with the envelope equation (1), demonstrating an excellent agreement. Then, we have performed an ideal experiment, by setting-up a Mach-Zender interferometer in Zemax, placing a plasma waveguide along the path of one arm. After running a Zemax simulation, so obtaining an interferogram (figure 4), we have analyzed the interferometric data to retrieve the electron plasma density from the plasma-induced phase shift. As shown in figure 6 , the result for the density profile retrieval is in perfect agreement with the values given as input to Zemax. Exploiting further its potentialities, Zemax can be used to test the feasibility of new geometries and material to be employed in the realization of plasma waveguides as well as to study their behaviour in specific experimental setups. In this sense, we have presented also the possibility to study the ray propagation in a curved capillary (figure 7), showing and modeling the laser beam guiding in a bent plasma channel. Furthemore, our model is not limited to parabolic plasma density profiles only, but it is possible to implement any sort of profile with an analytical description by realizing a proper DLL. Future studies will be devoted to the possibility of including other effects related to high intensity laser beams $\left(I>10^{18} \mathrm{~W} \mathrm{~cm}^{-2}\right)$, e.g. relativistic self-focusing and pump depletion due to the formation of high-amplitude plasma wakefields. 


\section{References}

[1] Wojda F et al 2009 Laser-driven plasma waves in capillary tubes Phys. Rev. E 80066403

[2] Andreev N E, Cros B, Gorbunov L M, Matthieussent G, Mora P and Ramazashvili R R 2002 Laser wakefield structure in a plasma column created in capillary tubes Phys. Plasmas 9 3999-4009

[3] Curcio A, Petrarca M, Giulietti D and Ferrario M 2016 Numerical and analytical models to study the laser-driven plasma perturbation in a dielectric gas-filled capillary waveguide Opt. Lett. 41 4233-6

[4] Nakanishi H et al 1991 Direct observation of plasma-lens effect Phys. Rev. Lett. 661870

[5] Pompili R et al 2017 Experimental characterization of active plasma lensing for electron beams Appl. Phys. Lett. 110104101

[6] Pompili R et al 2018 Focusing of high-brightness electron beams with active-plasma lenses Phys. Rev. Lett. 121174801

[7] Chiadroni E et al 2018 Overview of plasma lens experiments and recent results at sparc lab Nucl. Instrum. Methods Phys. Res. A 909 16-20

[8] Esarey E, Schroeder C B and Leemans W P 2009 Physics of laser-driven plasma-based electron accelerators Rev. Mod. Phys. 811229

[9] Bobrova N A, Esaulov A A, Sakai J-I, Sasorov P V, Spence D J, Butler A, Hooker S M and Bulanov S V 2001 Simulations of a hydrogen-filled capillary discharge waveguide Phys. Rev. E 65016407

[10] Hosokai T, Kando M, Dewa H, Kotaki H, Kondo S, Hasegawa N, Nakajima K and Horioka K 2000 Optical guidance of terawatt laser pulses by the implosion phase of a fast z-pinch discharge in a gas-filled capillary Opt. Lett. 25 10-2

[11] Butler A, Spence D J and Hooker S M 2002 Guiding of high-intensity laser pulses with a hydrogen-filled capillary discharge waveguide Phys. Rev. Lett. 89185003

[12] Andreev N E, Nishida Y and Yugami N 2002 Propagation of short intense laser pulses in gas-filled capillaries Phys. Rev. E 65056407

[13] Leemans W, Esarey E, Geddes C, Schroeder C and Tóth C 2006 Laser guiding for gev laser-plasma accelerators Phil. Trans. R. Soc. A 364 585-600

[14] Spence D J, Butler A and Hooker S M 2001 First demonstration of guiding of high-intensity laser pulses in a hydrogenfilled capillary discharge waveguide J. Phys. B: At. Mol. Opt. Phys. 344103

[15] Nakamura K, Nagler B, Tóth C, Geddes C G R, Schroeder C B, Esarey E, Leemans W P, Gonsalves A J and Hooker S M 2007 Gev electron beams from a centimeterscale channel guided laser wakefield accelerator Phys. Plasmas 14056708

[16] Kim H T, Pae K H, Cha H J, Kim I J, Yu T J, Sung J H, Lee S K, Jeong T M and Lee J 2013 Enhancement of electron energy to the multi-gev regime by a dual-stage laser-wakefield accelerator pumped by petawatt laser pulses Phys. Rev. Lett. 111165002

[17] Gonsalves A J et al 2019 Petawatt laser guiding and electron beam acceleration to $8 \mathrm{gev}$ in a laser-heated capillary discharge waveguide Phys. Rev. Lett. 122084801

[18] Karsch S et al $2007 \mathrm{Gev}$-scale electron acceleration in a gas-filled capillary discharge waveguide New J. Phys. 9415

[19] Curcio A and Petrarca M 2019 Diagnosing plasmas with wideband terahertz pulses Opt. Lett. 44 1011-4

[20] Gonsalves A J, Rowlands-Rees T P, Broks B H P, Van der Mullen J J A M and Hooker S M 2007 Transverse interferometry of a hydrogen-filled capillary discharge waveguide Phys. Rev. Lett. 98025002

[21] Brandi F and Gizzi L A 2019 Optical diagnostics for density measurement in high-quality laser-plasma electron accelerators High Power Laser Sci. Eng. 7 E26

[22] Bisesto F G et al 2018 The flame laser at sparc_lab Nucl. Instrum. Methods Phys. Res. A 909 452-5

[23] Costa G et al 2018 Characterization of self-injected electron beams from lwfa experiments at sparc_lab $\mathbf{N u c l}$. Instrum. Methods Phys. Res. A 909 118-22

[24] Bisesto F G, Castellano M, Chiadroni E and Cianchi A 2018 Zemax simulations describing collective effects in transition and diffraction radiation Opt. Express 26 5075-82

[25] Boné A, Lemos N, Figueira G and Dias J M 2016 Quantitative shadowgraphy for laser-plasma interactions J. Phys. D: Appl. Phys. 49155204

[26] Moore K 2017 Zemax opticstudio user manual Zemax LLC

[27] Rossi A R et al 2014 The external-injection experiment at the sparc_lab facility Nucl. Instrum. Methods Phys. Res. A 740 60-6

[28] Rossi A R et al 2016 Stability study for matching in laser driven plasma acceleration Nucl. Instrum. Methods Phys. Res. A 829 67-72

[29] Zigler A et al 2018 Consolidating multiple femtosecond lasers in coupled curved plasma capillaries Appl. Phys. Lett. 113183505

[30] Ehrlich Y, Cohen C, Zigler A, Krall J, Sprangle P and Esarey E 1996 Guiding of high intensity laser pulses in straight and curved plasma channel experiments Phys. Rev. Lett. 774186

[31] Luo J et al 2018 Multistage coupling of laser-wakefield accelerators with curved plasma channels Phys. Rev. Lett. 120154801

[32] Shiraishi S et al 2013 Laser red shifting based characterization of wakefield excitation in a laser-plasma accelerator Phys. Plasmas 20063103

[33] Reitsma A J W and Jaroszynski D A 2007 Propagation of a weakly nonlinear laser pulse in a curved plasma channel Phys. Plasmas 14053104

[34] Cros B, Courtois C, Matthieussent G, Di Bernardo A, Batani D, Andreev N and Kuznetsov S 2002 Eigenmodes for capillary tubes with dielectric walls and ultraintense laser pulse guiding Phys. Rev. E 65026405 